\title{
Discrimination of Different Types Damage of Tomato Seedling by Electronic Nose
}

\author{
Shao-Ming CHENG, Jun WANG ${ }^{\mathrm{a}}$, Yong-Wei WANG and Zhen-Bo WEI \\ College of Bio-systems Engineering and Food Science, Zhejiang University, Hangzhou, 310058, PR. China
}

\begin{abstract}
The profiles of volatile compounds emitted by plants varies in response to different damage.The potential of electronic nose technology to monitor such changes, with the aim of diagnosing plant health was investingted.An electronic nose(E-nose)was used to analyse tomato seedlings that were subjected to differnent types of damage(infection by Early blight disease, infection by Gray mold disease, mechanically damage, and undamaged). Principal component analysis(PCA), linear discrimination analysis(LDA), back-propagation neural network(BPNN), and support vector machine (SVM) network were used to evaluate the E-nose data.The results indicated that the E-nose can successfully discriminate between tomato seedling with different types of damage. The results of PCA and LDA showed that clusters of data were divided into 3 groups (ZP, HP, and CP/MP). Samples from groups $\mathrm{CP}$ and MP overlapped partially. Back-propagation neural network (BPNN) and support vector machine (SVM) network were used to evaluate the E-nose data. Good discrimination results were obtained using SVM and BPNN. The results demonstrate that it is plausible to use E-nose technology as a method for monitoring damage in tomato seedling.
\end{abstract}

\section{Introduction}

Plants increase the amount of volatile compounds that they release when they were damaged by insects or diseases. A number of studies have demonstrated that plants respond to damage with releasing a variety of volatiles [1-3]. Over the past decade, there has been significant progress in plant volatile research as a result of the increasing sensitivity of analytical instrumentation and improvements in molecular and biochemical analyses. However, this method involves several stages which makes it time consuming, especially at the stage of volatile-compound trapping and sample preparation. In addition, the technology is very expensive, the equipment is bulky, and the analysis requires skilled analysts. Therefore, it is not suitable for use in a warning system for plant insects and disease attacks in a commercial plant-growing setting.

An electronic nose (E-nose) system is sensor-based technology that measures total headspace volatiles and creates a unique smell print. An E-nose does not resolve the sample's volatiles into individual components but rather responds to the whole set of volatiles in a unique digital pattern. This pattern is the signature of a particular set of aromatic compounds. For each process or application of interest, a database of such digitized patterns is created, called the training set. When an unknown sample is exposed to the E-nose sensors, the E-nose first digitizes the sample's volatiles, and then

a Corresponding author: jwang@zju.edu.cn 
compares the resulting pattern with the existing training set. Its principle is different from that of chemical analysis methods such as gas and liquid chromatography, mass spectrometry, nuclear magnetic resonance, and spectrophotometry. The E-nose does not resolve the sample's volatiles into its individual components but responds to the whole set of volatiles in a unique digital pattern. E-nose technology has been successfully used in a variety of applications, including food quality and control [5,6,14], environmental monitoring [7,17,18], micro-organism identification (Magan et al., 2000), medical and health detection [15,16] and plant monitoring [9-13]. Recently, more research has been directed towards early detection of insect and disease attack. This technology has been used to differentiate volatile profiles from tomato, cucumber, and pepper leaves damaged by spider mites and beet armyworm $[4,8]$. The E-nose technology is rapid, sensitive, specific, non-destructive, and easy to use. Unlike GC-MS, the E-nose does not require prolonged pre-concentration intervals and is appealing as a potential technology for monitoring changes in disease-induced plant volatiles signatures. However, little information about E-nose monitoring of damaged tomato seedlings has been reported.

The objective of the present study was to determine the feasibility of detecting tomato seedling damage caused by the Early blight disease, the Gray mold disease, mechanical means damage and undamaged control seedlings utilizing an E-nose (PEN2). The aim was to develop discrimination models of E-nose signals for different types of damage. Principal component analysis (PCA), linear discrimination analysis (LDA), back-propagation neural network (BPNN) and support vector machine (SVM) network were used to verify the classification capacity of the proposed methods.

\section{Materials and Methods}

\subsection{Electronic nose}

In this study, a PEN2 (Airsense Analytics, Germany) E-nose, equipped with 10 different metal oxide semiconductor (MOS) sensors was used. The PEN2 system consists of a sampling apparatus, a detector unit containing the array of sensors, and pattern recognition software (WinMuster v.1.6) for data recording. The sensor array is composed of positioned in a small chamber. Each sensor has a certain degree of affinity towards specific chemicals or volatile compounds. Table 1 lists all the sensors used and their main application.

Table 1. Sensors used and their main application in PEN2

\begin{tabular}{|c|c|c|c|}
\hline $\begin{array}{l}\text { Number in } \\
\text { array }\end{array}$ & Sensor-name & General description & Reference \\
\hline S1 & W1C & Aromatic compounds & Toluene, $10 \mathrm{ppm}$ \\
\hline S2 & W5S & $\begin{array}{l}\text { Very sensitive, broad range sensitivity, react on nitrogene } \\
\text { oxides, very sensitive with negative signal }\end{array}$ & $\mathrm{NO}_{2}, 1 \mathrm{ppm}$ \\
\hline S3 & W3C & Ammonia, used as sensor for aromatic compounds & Propane, $1 \mathrm{ppm}$ \\
\hline S4 & W6C & Mainly hydrogen, selectively(breath gases) & $\mathrm{H}_{2}, 100 \mathrm{ppb}$ \\
\hline S5 & W5C & Alkanes, aromatic compounds, less polar compounds & Propane, $1 \mathrm{ppm}$ \\
\hline S6 & W1S & $\begin{array}{l}\text { Sensitive to methane (environment)ca.10ppm. Broad rang, } \\
\text { similar to No.8 }\end{array}$ & $\mathrm{CH}_{3}, 100 \mathrm{ppm}$ \\
\hline S7 & W1W & $\begin{array}{l}\text { Reacts on sulfur compounds, } \mathrm{H}_{2} \mathrm{~S} \quad 0.1 \mathrm{ppm} \text {. Otherwise } \\
\text { sensitive to many terpenes and sulfur organic compounds, } \\
\text { with are important for smell, limonene, pyrazine }\end{array}$ & $\mathrm{H}_{2} \mathrm{~S}, 1 \mathrm{ppm}$ \\
\hline S8 & W2S & $\begin{array}{l}\text { Detects alcohol's, partially aromatic compounds, broad } \\
\text { range }\end{array}$ & $\mathrm{CO}, 100 \mathrm{ppm}$ \\
\hline S9 & W2W & Aromatic compounds, sulfur organic compounds & $\mathrm{H}_{2} \mathrm{~S}, 1 \mathrm{ppm}$ \\
\hline S10 & W3S & $\begin{array}{l}\text { Reacts on high concentrations }>100 \mathrm{ppm} \text {,sometime very } \\
\text { selective(methane) }\end{array}$ & $\begin{array}{l}\mathrm{H}_{2} \mathrm{~S}, 1 \mathrm{ppm} \\
\mathrm{CH}_{3}, 10 \\
\mathrm{CH}_{3}, 100 \mathrm{ppm}\end{array}$ \\
\hline
\end{tabular}


Figure.1 shows a schematic diagram of the PEN2 E-nose measurements during the experiments.Each tomato seedling was enclosed in a cylindrical stainless steel support $(400 \mathrm{~mm}$ high $\times 100 \mathrm{~mm}$ internal diameter) covered with a plastic bag over the top.The plastic bag was a colourless and odourless food-grade polyethylene bag $(0.08 \mathrm{~mm}$ thick $)$. The plant was sealed at the lower stem by 2 hermetic boards with a hole in the middle.This excluded the ordour coming from the medium. The plant was kept at room temperature $\left(28 \pm 2^{\circ} \mathrm{C}\right)$ for $20 \mathrm{~min}$ before static headspace sampling was begun.

Before each measurement, the E-nose system was cleaned with zero-air(air filtered on active carbon).The main purpose of this was to clean the circuit and to return the sensors to their baselines.During the measurement,the headspace gas of a plant was pumped into the sensor chamber at a constantrate of $200 \mathrm{ml} \mathrm{min}-1$ through a Teflon tube $(3 \mathrm{~mm})$ connected to a needle. When the headspace gas that had accumulated in the bag was pumped into the sensor chamber,the ratio of conductance of each sensor changed.The response of each sensor was expressed as a ratio of conductance $(\mathrm{G} / \mathrm{G} 0 ; \mathrm{G}$ and $\mathrm{G} 0$ are the respective conductivities of the sensors when the seedling gas and the zero gas pass over).The measuement procedure was controlled by a computer program. The measurement time was $65 \mathrm{~s}$, which was sufficient for the sensors to reach stable values. The interval for data collection was 1s.A conputer recorded the response of the E-nose every second.The fulsh time was set to $40 \mathrm{~s}$. When the measurement was completed, the acquered data was stored for later use.

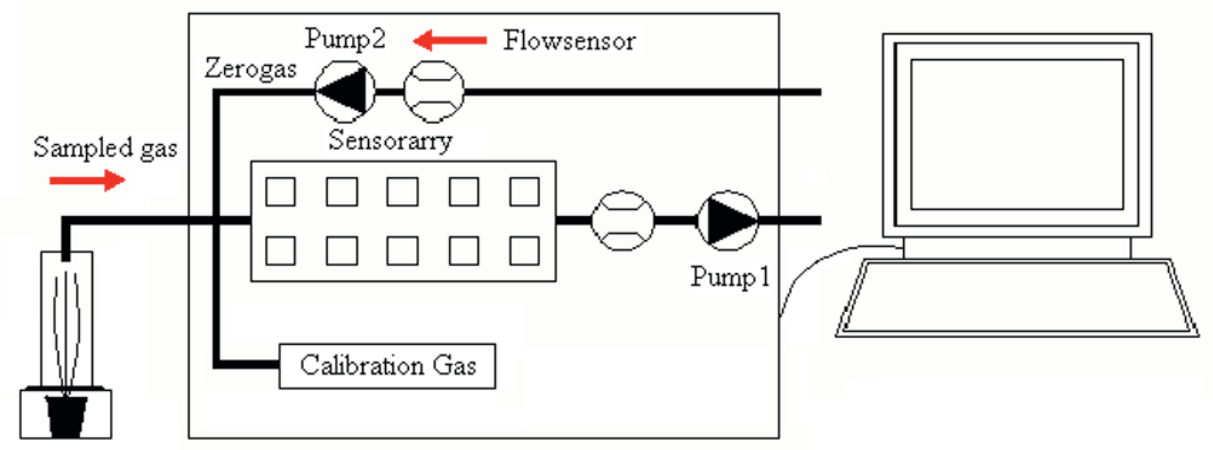

Figure 1. Schematic diagram of the electronic nose measurements of PEN2

\subsection{Tomato seedlings}

The variety of tomato seedling was ZheZa 809. Pre-germinated seeds were sown at common clay pots ( $80 \mathrm{~mm}$ diameter and $100 \mathrm{~mm}$ high) in greenhouse where the temperature was maintained at $28 \pm 2^{\circ} \mathrm{C}$ and relative humidity $(\mathrm{RH})$ was $70-80 \%$. Plants were managed daily. After 35 days, uniform-tomato seedlings were selected for the experiment.

\subsection{Plant treatment}

In this study, 4 treatments were tested: (1)damage caused by Early blight disease(EP),(2)damage caused by Gray mold disease(GP),(3)mechanically damage(MP), and(4)undamaged(CP).

First the Pathogenic bacteria of Early blight disease and Gray mold disease were training at laboratory, and then the diseases were vaccinated to 35 day tomaot seedlings, respectively. The vaccinated tomato seedlings were kept with high temperature and high $\mathrm{RH}$ for $48 \mathrm{~h}$.

For the Early blight disease treatment, 4 leaves disease infection were used per tomato seedling. For the Gray mold disease, also 4 leaves disease infection were used per tomato seedling. For the mechanical damage treatment, a single tomato seedling was punched 90 times with a 
needle $(0.8 \times 26 \mathrm{~mm})$.Sixty-four plants were sellected for the experiment,and there were 16 replicates for each treatment.

\subsection{Data analysis}

In this study, the data was subjected to different statistical analyses,including Principal component analysis (PCA), linear discrimination analysis(LDA), back-propagation neural network(BPNN), and support vector machine (SVM) network.All statistical analyses were conducted with SAS v.8 and Matlab 7.0.

\section{Results and discussion}

\subsection{Electronic nose responses to tomato seedling volatiles}

Figure. 2 shows the typical responses of 10 sensors during measurements on a tomato seedling. Each curve represents the variation in conductivity of each sensor with time. Electro-value changes when the volatiles from the tomato seedling reach the measurement chamber. The response signal was measured and expressed as, where G and G0 are the resistance of a sensor in clean air and in a detecting gas, respectively. It is clear that the ratio of G/G0 was close to 1.0 at the initial period, then increased or decreased gradually,and stabilized after about 60s. The signals of each sensor at response $60 \mathrm{~s}$ were used in the subsequent analyses.

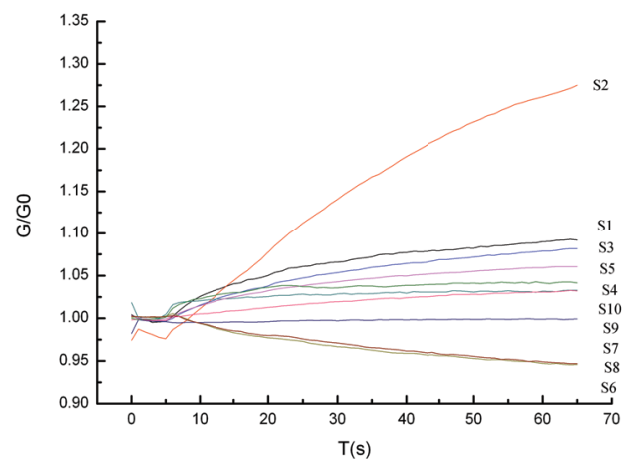

(a) Control tomato seedling

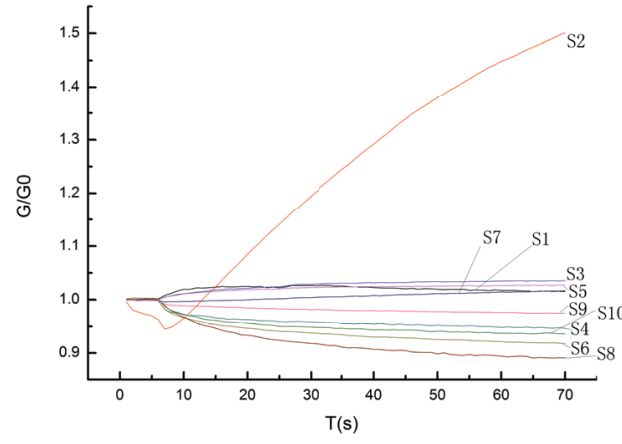

(c) Early blight disease damage tomato seedling

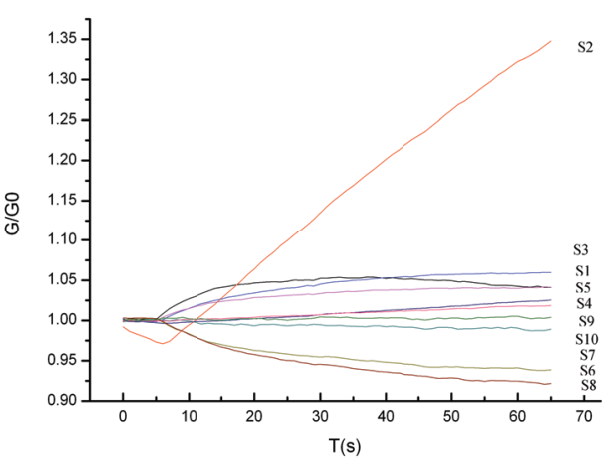

(b) mechanical damage tomato seedling

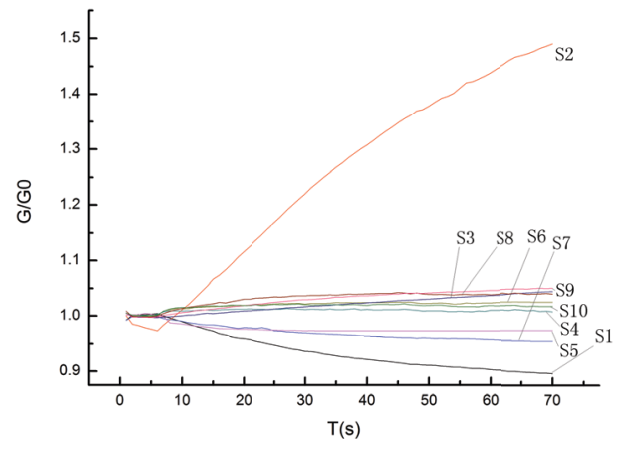

(d) Gray mold disease damage tomato seedling

Figure 2. Response curve of 10 sensors to tomato seedling volatiles 


\subsection{Signal analysis}

Figure. 3 shows the changes of the sensor signals in response to tomato seedling with different types of damage. Each point represents the mean value of each sensor's response signal to tomato seedlings, linked to the measurements of conductantce increases or decreases experienced by the sensors.The E-nose sensor response changed by tomato seedling with 4 different types of treatments.The values of the sensor response singnals differed with the different types of damange.The variation in the sensor responses in the $\mathrm{CP}$ was small compared to the 3 groups of damaged tomato seedlings(Figure.3).This result is due to the specific changes in the chemical composition in tomato seedlings after the damage.

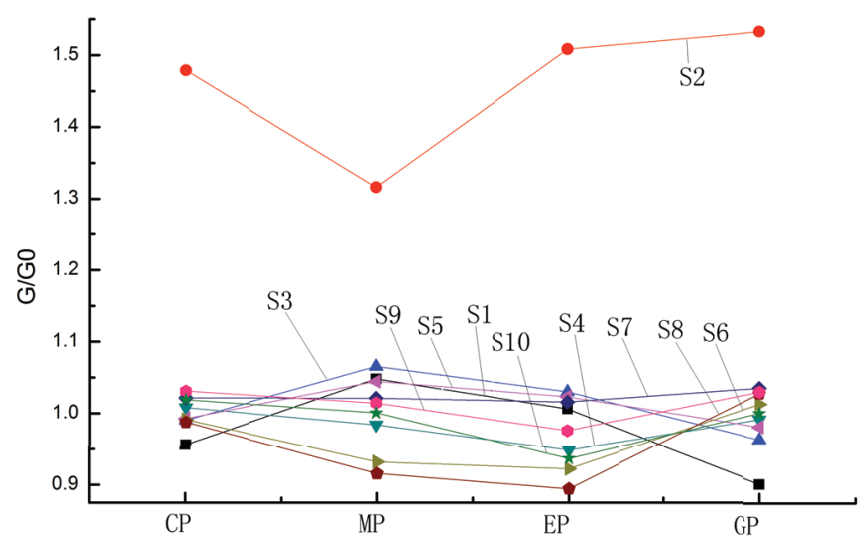

Figure 3. Value of each sensor's response to tomato seedling with different types of damage (CP, control seedlings; MP, mechanically damaged seedlings; EP, seedlings infected by Early blight disease; GP,seedlings infected by Gray mold disease)

\subsection{PCA and LDA analysis}

PCA and LDA are commonly used techniques for data classification and dimensionality reduction. The data from 64 samples (16 samples from each group) obtained by the E-nose for 4 tomato seedling groups were used for the PCA.Fig.4 shows the results of the PCA. The first component contributed $59.93 \%$ of the total variance; the second component, $33.01 \%$. This added up to $92.94 \%$ in total. In Fig.4, clusters of data were divided into 3 groups (CP/MP, EP and GP).Samples from groups EP and GP were easily discriminated, whereas samples from groups CP and MP overlapped partially. PCA did not clearly discriminate between these different types of tomato seedlings. Therefore, LDA was used for further separation of the tomato seedling samples.

The results of the LDA are shown in Figure.5. Function1(LD1) contributed $85.83 \%$ of the total variance; Fuction2(LD2), $10.52 \%$, adding up to a total of $96.35 \%$.In the LDA plot, 4 groups of tomato seedling samples could be clearly distinguished. The separation of the samples was better using LDA than using PCA. 


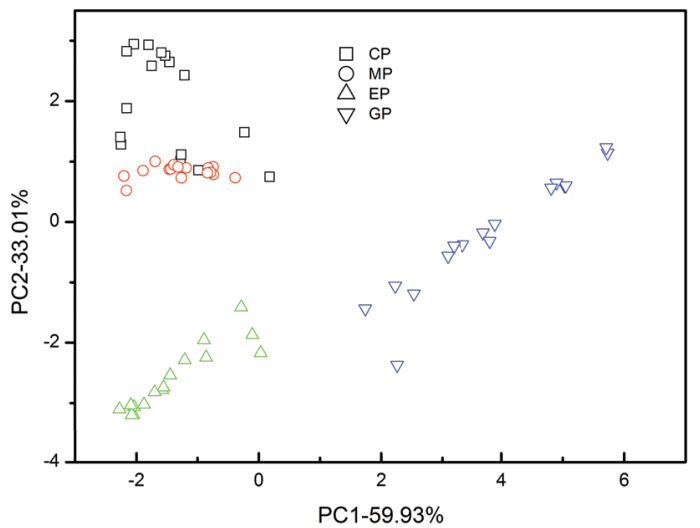

Figure 4. PCA plot for tomato seedlings with different types of damage (CP, control seedlings; MP, mechanically damaged seedlings; EP, seedlings infected by Early blight disease; GP,seedlings infected by Gray mold disease)

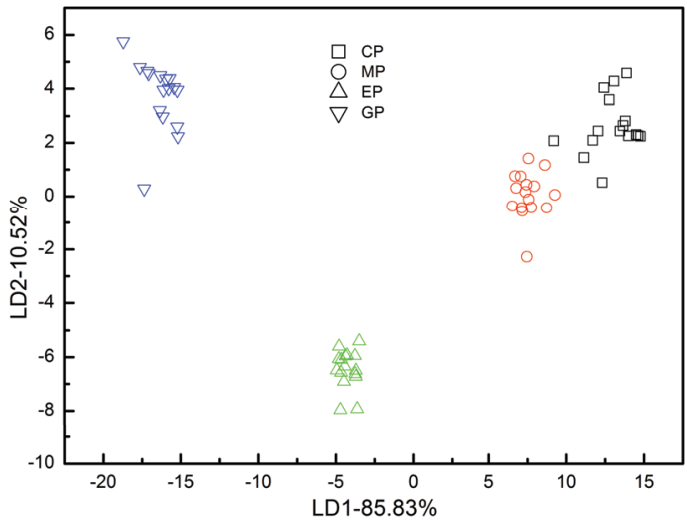

Figure 5. LDA plot for tomato seedlings with different types of damage (CP, control seedlings; MP, mechanically damaged seedlings; EP, seedlings infected by Early blight disease; GP,seedlings infected by Gray mold disease)

\subsection{BPNN and SVM}

In this study, sixty-four samples (16 duplicates for each of the 4 groups) were divided into 2 groups: 40 samples (10 samples of each group) for the training set and 24 samples (6 samples of each group) for testing set.

For the BPNN, the chosen architecture of the artificial neural network was a 10-15-1 three-layer back-propagation according to Kolmogorov's theorem. The 10 input neurons correspond to the data obtained by 10 sensors, while the 1 output is the different types of damage. The training function is 'traingda' and the training epoch is 2000.The training parameters were chosen a epoch of 0.01 and a goal of 0.001 .The result is shown in Table 2.The correct rate of the training set was $100 \%$. The correct rates of the testing set for the CP, MP, EP and GP groups were $100 \%, 100 \%, 83.3 \%$, and $100 \%$, respectively.

The SVM network also had 10 and 1 neurons in the input and output layers, respectively. The 
kernel function used in this paper was 'radial basis kernel function'. The SVM analysis showed an overall classification success of $100 \%$ for the training sets and $95.8 \%$ for the testing sets. The result is shown in Table 2.The results indicate that it is possible to use E-nose signals to discriminate between tomato seedlings with different types of damage.

Table 2. Results of BPNN and SVM analyses for tomato seedling with different types of damage

\begin{tabular}{cccccc}
\hline \multirow{2}{*}{ Network style } & $\begin{array}{c}\text { Correct rate of } \\
\text { training set }\end{array}$ & CP & MP & EP & GP \\
\hline BPNN & $100 \%$ & $100 \%$ & $100 \%$ & $83.3 \%$ & $100 \%$ \\
SVM & $100 \%$ & $100 \%$ & $100 \%$ & $83.3 \%$ & $100 \%$ \\
\hline
\end{tabular}

\section{Conclusions}

To sum up, the results prove that the E-nose PEN 2 can successfully distinguish between tomato seedlings with different types of damage (infection by Early blight disease, infection by Gray mold disease, mechanically damage, and undamaged). The LDA discriminates more effectively between the different groups than the PCA. Good discrimination results are obtained in BPNN and SVM. The results demonstrate that it is plausible to use E-nose technology as a method for monitoring damage in tomato seedling.

\section{Acknowledgement}

The first author acknowledges the financial grant from the National Natural Science Foundation of China (Grant No. 31370555).

\section{References}

1. Kessler, I.T.Baldwin.Defensive function of herbivore-induced plant volatile emissions in nature. Science, 291(2001):2141-2144

2. Y.J.Lu,X.Wang,Y.G.Lou,J.A.Cheng.Role of ethylene signaling in the production of rice volatiles induced by the rice brown planthopper Nilaparvata lugens, Chines Science Bulletin, 51(2006):2457-2465

3. Y.G. Lou, X.Y. Hua, T.C.J.Turlings, J.A.Cheng, X.X.Chen, G.Y.Ye.Differences in induced volatile emissions among rice varieties result in differential attraction and parasitism of Nilaparvata lugens eggs by the parasitoid Anagrus nilaparvatae in the field, Journal of Chemical Ecology,32(2006):2375-2387

4. J.Laothawornkitkul,J.P.Moore,J.E.Taylor,M.Possell,T.D.Gibson,C.N.Hewitt,N.D.Paul.Discrimina tion of plant volatile signatures by an electronic nose: a potential technology for plant pest and disease monitoring, Environmental Science and Technology, 42(2008):8433-8439

5. A.H.Gomez,J.Wang,G.X.Hu,A.G.Perira.Discrimination of storage shelf-life for mandarin by electronic nose technique, LWT-Food Science and Technology,40(2007):681-689

6. L.Pang,J.Wang,X.Lu,H.Yu.Discrimination of storage age for wheat by E-nose, Transactions of the ASABE,51(2008):1707-1712

7. A.C. Bastos,N.Magan.Potential of an electronic nose for the early detection and differentiation between Stretomyces in potable water, Sensors and Actuators B:Chemical,116(2006):151-155

8. N.Magan,P.Evans.Volatiles as indicator of fungal activity and differentiation between species, and the potential use of electronic nose technology for early detection of grain spoilage, Journal of Stored Products Research,36(2000):319-340 
9. M. Kunert,A.Biedermann,T.Koch,W.Boland.Ultrafast sampling and analysis of plant volatiles by a hand-held miniaturized GC with pre-concentration unit: kinetic and quantitative aspects of plant volatile production, Journal of Separation Science,25(2002):677-684

10. R.M.Jansen,J.W.Hofstee,J.Wildt,F.W.Verstappen,H.J.Bouwmeester,E.J.vahenten.Induced plant volatiles allow sensitive monitoring of plant health status in greenhouse, Plant Signal Behav.,4(2009):824-829

11. H.M.Zhang,J.Wang.Detection of age and insect damage incurred by wheat with an electronic nose, Journal of Stored Products Research,43(2007):489-495

12. W.G.Henderson,A.Khalilian,Y.J.Han,J.K.Greeme,D.C.Degemjardt.Detecting stink bugs/damage in cotton utilizing a portable electronic nose, Computers and Electronics in Agriculture, 70(2010): 157-162

13. B.Zhou,J.Wang.Use of electronic nose technology for identifying rice infestation by Nilaparvata lugens, Sensors and Actuators B:Chemical,160(2011):15-21

14. S.Cheng,Y.Wang,J.Wang,Z.Wei,Q.Lu.Detection of eggshell crack using electronic nose[J]. Transactions of the ASABE, 53(2010):789-794

15. J.Dummer,M.Storer,M.Swanney,M.McEwan,A.S.Thomas,S.Bhandari,S.Chambers,R.Dweik,M.E pton.Analysis of biogenic volatile organic compounds in human health and disease,Trends in Analytical Chemistry,30(2011):960-967

16. M.Bernabei,G.Pennazza,M.Santonico,C.Corsi,C.Roscioni,R.Paoeesse,C.DiNatale,A.D'Amico.A preliminary study on the possibility to diagnose urinary tract cancers by an electronic nose, Sensors and Actuators B:Chemical,131(2008):1-4

17. J.H.Sohn,N.Hudson,E.Gallagher,M.Dunlop,L.Zeller,M.Atzeni.Implementation of an electronic nose for continuous odour monitoring in a poultry shed, Sensors and Actuators B:Chemical, 133(2008):60-69

18. S.DeVito,E.Massera,M.Piga,L.Martinotto,G.DiFrancia.On field calibration of an electronic nose for benzene estimation in an urban pollution monitoring scenario, Sensors and Actuators B:Chemical, 129(2008):750-757 